\title{
Optic neuritis following SARS-CoV-2 infection
}

\author{
Mónica Saray Rodríguez-Rodríguez ${ }^{1}$ (D - Rosa María Romero-Castro ${ }^{1}$ - Claudia Alvarado-de la Barrera ${ }^{1}$. \\ María Gabriela González-Cannata ${ }^{1}$. Ana Karen García-Morales ${ }^{1}$ Santiago Ávila-Ríos ${ }^{1}$
}

Received: 20 September 2020 / Revised: 24 January 2021 / Accepted: 4 February 2021 / Published online: 23 March 2021

(c) Journal of NeuroVirology, Inc. 2021

\begin{abstract}
The most common neurologic symptoms in COVID-19 are headache, anosmia, and dysgeusia. Optic neuritis is an unusual manifestation of SARS-CoV-2 infection. We report a case of a patient who initially consulted for vision loss in the absence of respiratory symptoms. She was diagnosed with optic neuritis following SARS-CoV-2 infection. Delay in diagnosis of neuro-ophthalmic manifestations of COVID-19 may lead to irreversible optic atrophy. A mechanism in which viral antigens would induce an immune response against self-proteins, or direct SARS Cov-2 infection of the central nervous system, may be involved in optic nerve injury.
\end{abstract}

Keywords Optic neuritis · COVID-19 $\cdot$ SARS-CoV-2 $\cdot$ Demyelinating disease

\section{Introduction}

The coronavirus disease (COVID)-19 is caused by the novel severe acute respiratory syndrome coronavirus 2 (SARS-CoV-2) (Coronaviridae Study Group of the International Committee on Taxonomy of Viruses 2020) and has a predominant clinical presentation with respiratory disease. However, a number of patients have experienced neurological manifestations such as headache, anosmia, ageusia, stroke, Guillain-Barré syndrome, impaired consciousness, coma, seizure, and encephalopathy (Zubair et al. 2020). In addition, growing literature documents cases of new onset neuro-ophthalmic disease in the context of SARS-CoV-2 infection, including optic neuritis (ON) (Zhou et al. 2020), Miller Fisher syndrome (Dinkin et al. 2020), cranial neuropathies (Gutierrez-Ortiz et al. 2020), vision loss (Cyr et al. 2020), and neuromyelitis optica spectrum disorder (Shaw et al. 2020).

Mónica Saray Rodríguez-Rodríguez and Rosa María RomeroCastro equally contributed to this work.

Mónica Saray Rodríguez-Rodríguez monica.rodriguez@cieni.org.mx

1 Centro de Investigación en Enfermedades Infecciosas, Instituto Nacional de Enfermedades Respiratorias Ismael Cosío Villegas, Calzada de Tlalpan 4502, Col. Sección XVI, CP , 14080 Ciudad de Mexico, Mexico
We report a case of a patient who initially consulted for ocular symptoms and was diagnosed with $\mathrm{ON}$ following SARS-CoV-2 infection.

\section{Case description}

A 55-year-old woman consulted on April 25, 2020, for a 12-day history of persistent headache and left eye pain exacerbated with eye movements, refractory to treatment with non-steroidal anti-inflammatory drugs. She complained of unilateral, gradual visual loss, decreasing visual acuity, and chromatic impairment. She had no comorbidities or associated systemic symptoms. Direct patient interrogation excluded personal and family history of demyelinating or autoimmune diseases, unexplained blindness, and Leber's hereditary optic neuropathy. Blood testing indicated absence of vitamin B12 deficiencies or other nutritional optic neuropathy. Clinical examination revealed decreased oxygen saturation (SpO2 88\%) and the SARS-CoV-2 realtime RT-PCR test from nasopharyngeal swab was positive. She had no respiratory symptoms, and chest computed tomography showed bilateral ground-glass opacities suggestive of viral pneumonia. On examination, the right eye visual acuity (VA) was 20/40 and left eye VA was 20/200, with a relative afferent pupillary defect in the left eye. Optic disc and optical coherence tomography (OCT) were normal. Automatized visual field showed inferior centrocecal and 
centronasal scotomas in the left eye (Fig. 1). The patient received ambulatory treatment with supplementary oxygen and $1 \mathrm{~g} /$ day of intravenous methylprednisolone for 5 days, followed by an oral prednisone taper.

One month later, right eye VA was 20/40 and left eye VA was 20/400. She had a relative afferent pupillary defect, significant optic disc pallor and a centrocecal scotoma. OCT indicated nerve fiber layer thinning in the temporal sector ( $1 \%$ normal deviation), peripapillary retinal nerve fiber loss and ganglion cell axon decrease in the left eye (Fig. 2). Magnetic resonance imaging (MRI) of the brain indicated absence of lesions. MRI of the orbit showed mild increased thickness and signal in the left optic nerve (Fig. 3). Laboratory results showed the presence of oligoclonal IgG bands in cerebrospinal fluid (CSF) but not in serum. Antiaquaporin-4 IgG antibodies were not detected in CSF nor in serum. Viral and bacterial CSF cultures and the SARSCoV-2 RNA PCR were negative. Serum levels of inflammatory markers were within normal ranges.

At a follow-up visit 3 months later, ocular pain decreased. Nevertheless, left eye vision did not improve, so optic atrophy remained despite treatment.
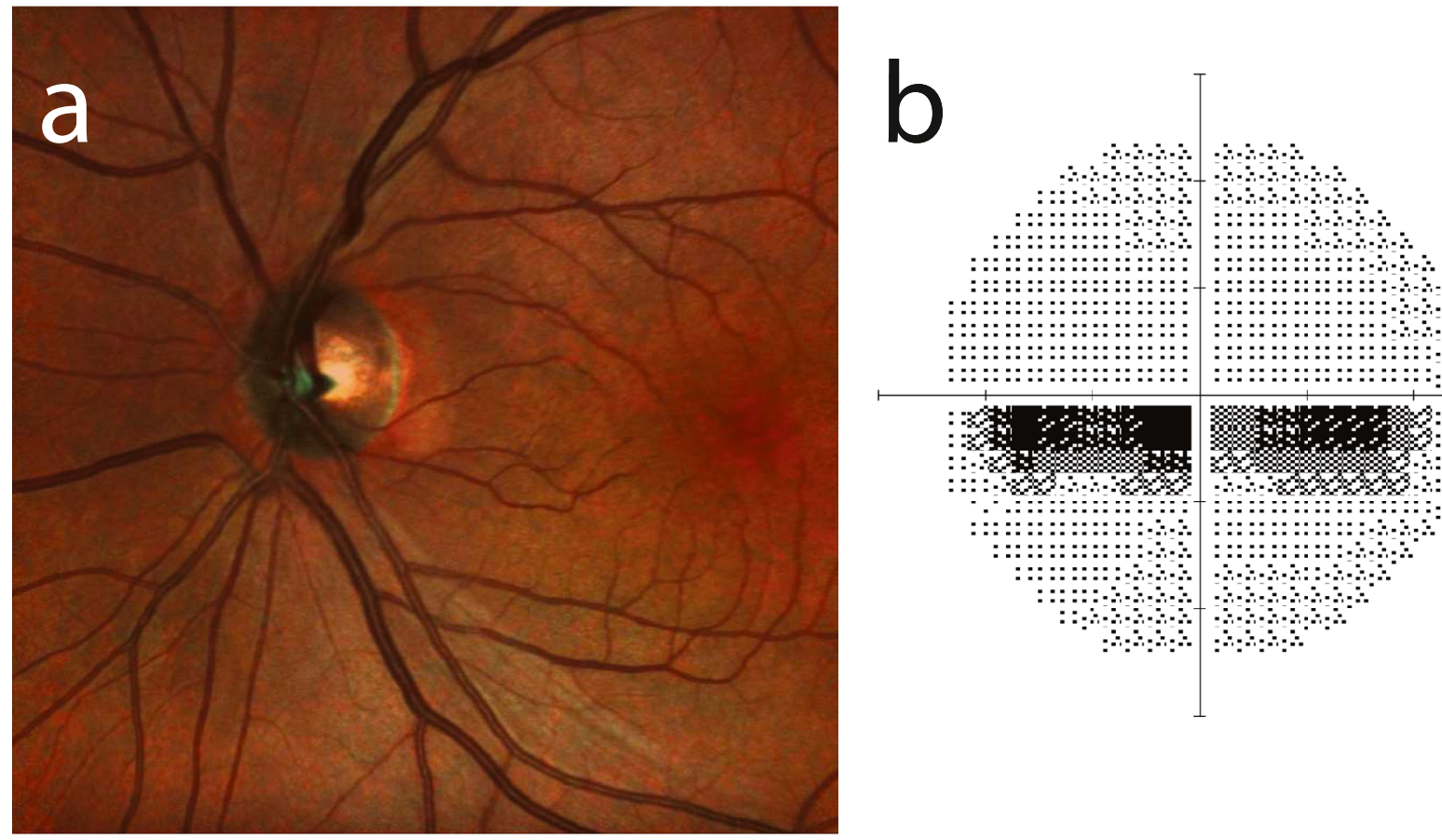

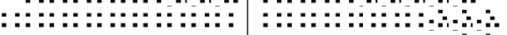

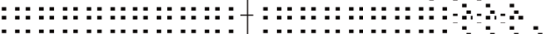

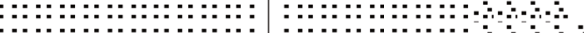

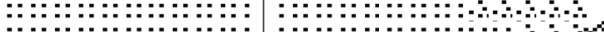

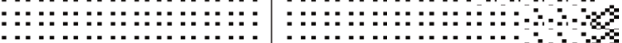

$:::::::::::::::::::::$

$: 3$

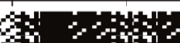

$:::::::::::::::: 9:-8 \%$

:

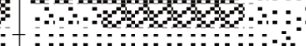

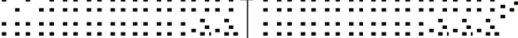

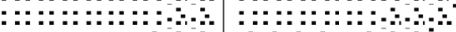

a

$a$

$\therefore \leftrightarrow$
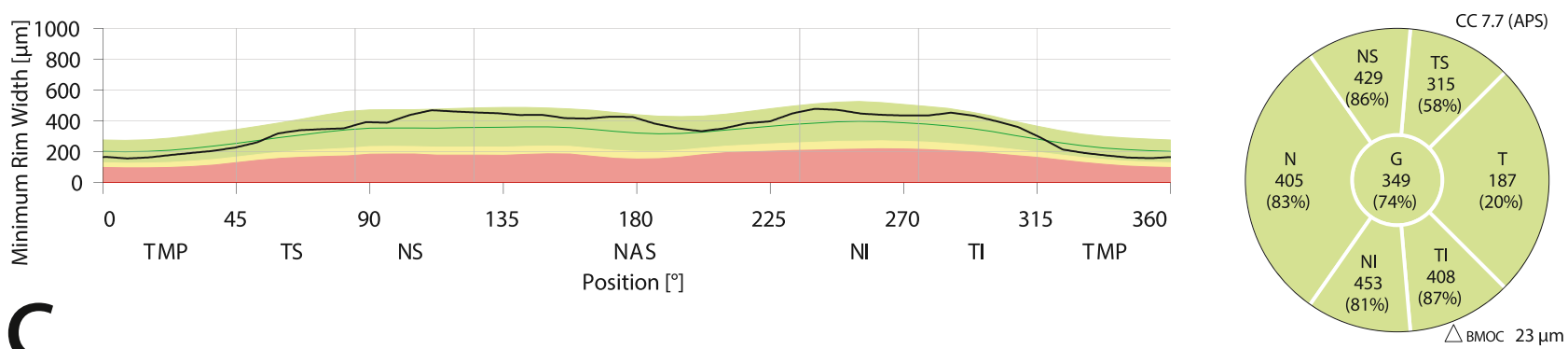

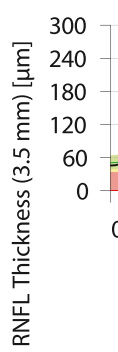
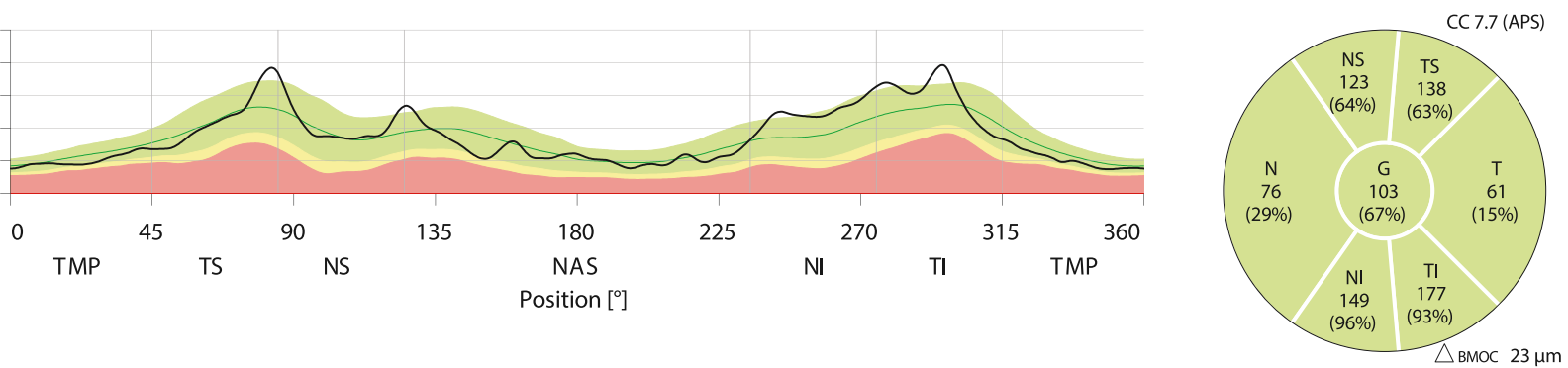

Fig. 1 Initial color fundus photographs, visual field test, and optical coherence tomography (OCT) of the left eye. a Normal optic disc. b Automatized visual field showing inferior centrocecal and centronasal scotomas. $\mathbf{c}$ Normal spectral-domain OCT 

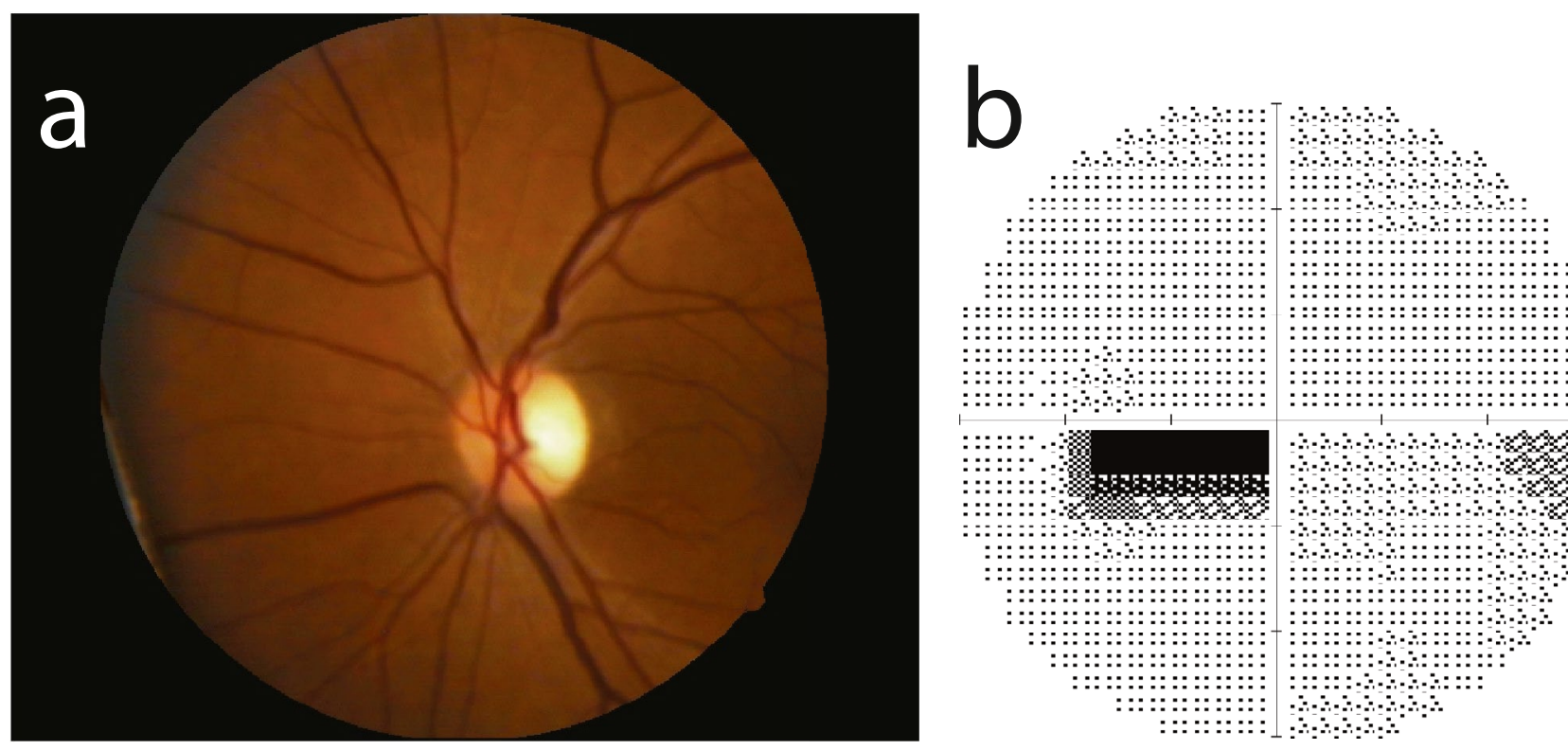

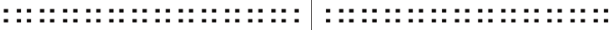
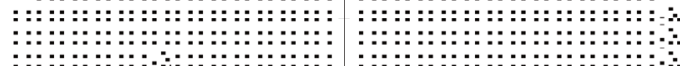

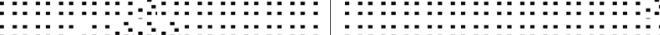
:::: : ::::::::: ::::::::::::::::::::::1

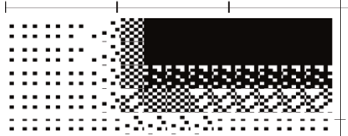

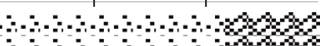
$04840 \%$

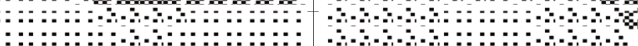

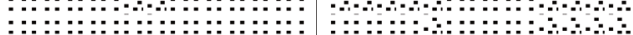

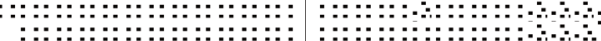

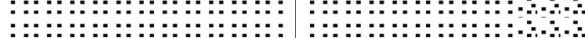

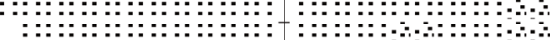

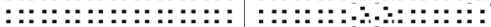

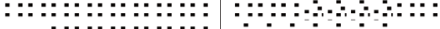

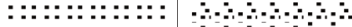
:::::::: $:-1 ;$
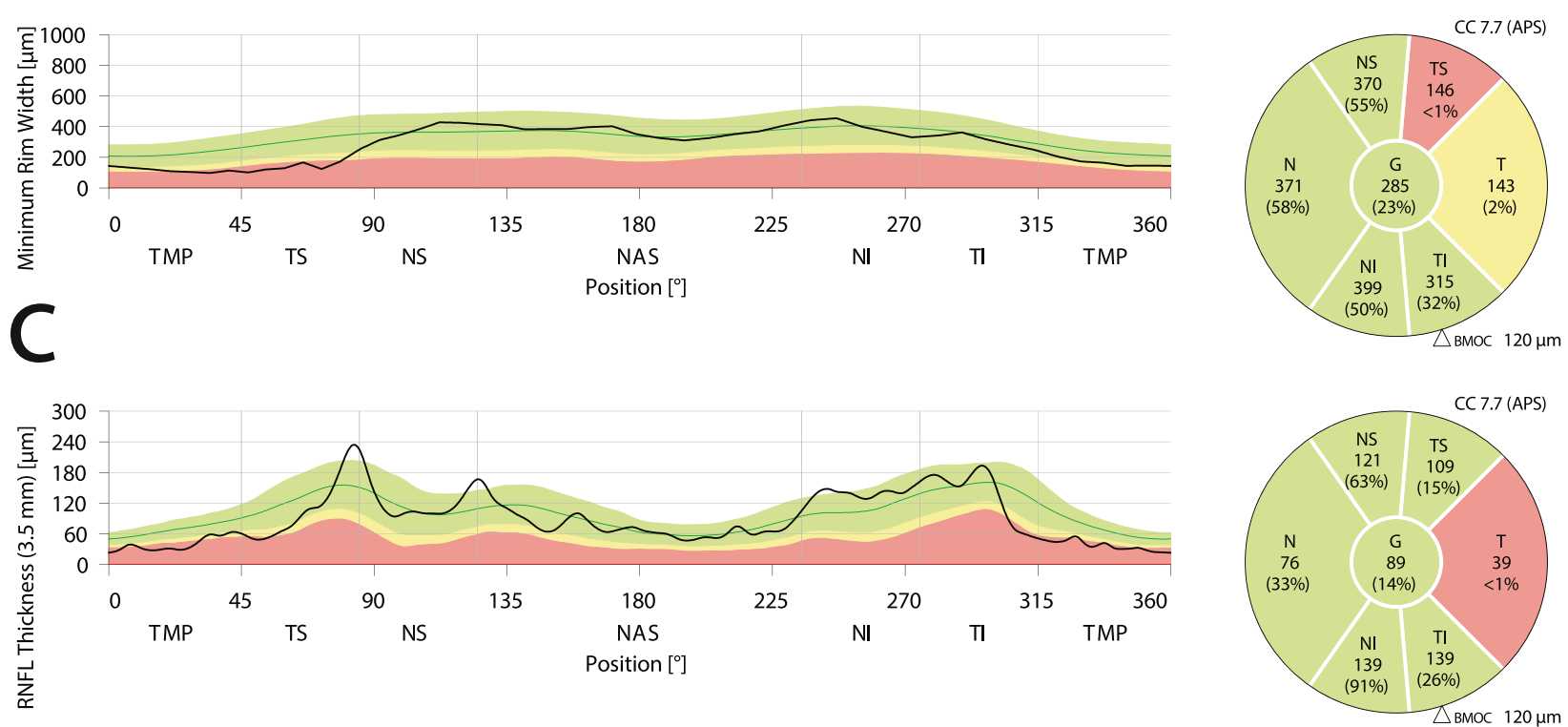

Fig. 2 Color fundus photographs, visual field test, and optical coherence tomography (OCT) of the left eye. a Optic disc pallor. b Automatized visual field showing a centrocecal scotoma. c OCT indicated

\section{Discussion}

We report a case of vision loss associated with unilateral $\mathrm{ON}$, as initial presentation of SARS-CoV-2 infection. The presence of oligoclonal $\mathrm{IgG}$ bands in CSF is supportive evidence of central nervous system (CNS) inflammation, multiple sclerosis, or infection. SARS-CoV-2 may enter the brain through the olfactory bulb, carriage across the blood-brain barrier following viraemia, or via infected leukocytes (Ellul et al. 2020). SARS-CoV-2 infection nerve fiber layer thinning in the temporal sector (1\% normal deviation), peripapillary retinal nerve fiber loss and ganglion cell axon decrease

occurs via the angiotensin-converting enzyme 2 (ACE2) receptor, expressed in CNS cells (Lukiw et al. 2020). The negative CSF SARS-CoV-2 PCR result does not rule out the possibility of direct CNS infection because CSF SARSCoV-2 PCR testing has not been validated in clinical settings. In this context, optic atrophy could also have been caused by a small capillary ischemic event associated with endothelitis or microthrombosis, since SARS-CoV-2 infection has been associated with prothrombotic effects and papillophlebitis (Insausti-García et al. 2020). Alternatively, 


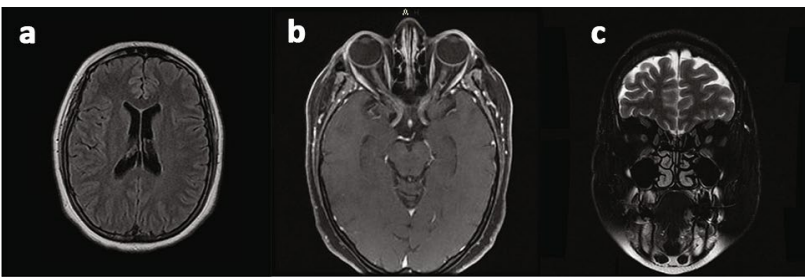

Fig. 3 Magnetic resonance imaging (MRI). a Brain MRI, T2 FLAIR axial image showing absence of lesions. b Orbit MRI, STIR T1 contrast axial image without enhancement in optic nerve. c Orbit MRI, STIR T2 coronal image showing mild increased thickness and signal in the left optic nerve

this could be a case of parainfectious or postinfectious demyelinating condition linked to a prodromal viral illness, because CSF cultures were negative for other pathogens and there were no brain lesions indicative of multiple sclerosis. A mechanism of molecular mimicry in which viral antigens would induce an immune response against self-proteins, may be responsible for tissue injury. In fact, the potential of SARS-CoV-2 for autoantibody production was recently illustrated by a case report of SARS-CoV-2 infection followed by myelin oligodendrocyte glycoprotein (MOG)-IgG-related ON and myelitis (Zhou et al. 2020). It is worth to mention that common findings in MOG-IgGrelated $\mathrm{ON}$ include bilateral, longitudinally extensive lesions in the retrobulbar optic nerve and spinal cord (Akaishi et al. 2016) and perineural optic nerve enhancement associated with disc edema (Kim et al. 2015). Considering that none of these features were observed in our patient, and oligoclonal bands are uncommon in MOG-IgG-related-ON, testing of MOG-IgG antibodies was deemed unnecessary. However, since we are still learning about new manifestations of MOG-IgG-related-ON and isolated ON is not rare with MOG-IgG antibodies (Ramanathan et al. 2018), there is a remote possibility that the patient could have this condition.

A case of multiple sclerosis following SARS-CoV-2 infection was also reported in a patient with inflammation in the right optic nerve and demyelinating lesions in the CNS (Palao et al. 2020). There is another reported case of unilateral panuveitis and $\mathrm{ON}$ as the first manifestation of SARS-CoV-2 infection, before the onset of pulmonary symptoms (Benito-Pascual et al. 2020). It remains to be elucidated if SARS-CoV-2 is associated with triggering or exacerbating inflammatory processes leading to demyelinating disease. However, the association of neurologic injury with a virus of the Coronaviridae family is not new. Guillain-Barré syndrome, and acute motor axonal neuropathy variants, were documented in the context of SARS-CoV-1 infection (Tsai et al. 2005).

Despite ON is mainly idiopathic, it may be related to demyelinating lesions, autoimmune disease, infectious/ parainfectious causes, and inflammatory/postvaccination immune responses. The classic presentation of $\mathrm{ON}$ associated with multiple sclerosis is unilateral, moderate, with painful vision loss, afferent pupillary defect and normal fundus examination. In consequence, we consider that our patient could have the potential of developing demyelinating lesions leading to multiple sclerosis, which would need to be defined in future follow-up visits.

\section{Conclusion}

Ocular symptoms are uncommon in COVD-19 and may represent the prodromal signs of the disease. Thus, we suggest that SARS-CoV-2 infection should be suspected in patients with ocular manifestations, even in absence of respiratory symptoms. Failure to diagnose neuro-ophthalmic manifestations of SARS-CoV-2 infection may lead to delayed treatment, further ocular injury and irreversible vision loss.

Author contribution Conceptualization: Mónica Saray RodríguezRodríguez; data curation: Mónica Saray Rodríguez-Rodríguez, Claudia Alvarado-de la Barrera; funding acquisition: Santiago Ávila-Ríos; Investigation: Mónica Saray Rodríguez-Rodríguez; Rosa María Romero-Castro; María Gabriela González-Cannata; project administration: Mónica Saray Rodríguez-Rodríguez; validation: Mónica Saray Rodríguez-Rodríguez; Ana Karen García-Morales; visualization: Claudia Alvarado-de la Barrera; Mónica Saray Rodríguez-Rodríguez; Ana Karen García-Morales; writing-original draft preparation: Mónica Saray Rodríguez-Rodríguez; writing—review and editing: Claudia Alvarado-de la Barrera; Mónica Saray Rodríguez-Rodríguez. All authors have approved the manuscript, and they have contributed significantly to the work.

\section{Declarations}

Conflict of interest The authors declare that they have no conflict of interest.

\section{References}

Akaishi T, Sato DK, Nakashima I, Takeshita T, Takahashi T, Doi H, Kurosawa K, Kaneko K, Kuroda H, Nishiyama S, Misu T, Nakazawa T, Fujihara K, Aoki M (2016) MRI and retinal abnormalities in isolated optic neuritis with myelin oligodendrocyte glycoprotein and aquaporin-4 antibodies: a comparative study. J Neurol Neurosurg Psychiatry 87:446-448. https://doi.org/10.1136/jnnp-2014-310206

Benito-Pascual B, Gegúndez JA, Díaz-Valle D, Arriola-Villalobos P, Carreño E, Culebras E, Rodríguez-Avial I, Benítez-Del Castillo JM (2020) Panuveitis and optic neuritis as a possible initial presentation of the novel coronavirus disease 2019 (COVID-19). Ocul Immunol Inflamm 28:922-925. https://doi. org/10.1080/09273948.2020.1792512

Coronaviridae Study Group of the International Committee on Taxonomy of Viruses (2020) The species Severe acute respiratory syndrome-related coronavirus: classifying 2019-nCoV and 
naming it SARS-CoV-2. Nat Microbiol 5:536-544. https://doi. org/10.1038/s41564-020-0695-z

Cyr DG, Vicidomini CM, Siu NY, Elmann SE (2020) Severe bilateral vision loss in 2 patients with coronavirus disease 2019. J Neuroophthalmol 40:403-405. https://doi.org/10.1097/ WNO.0000000000001039

Dinkin M, Gao V, Kahan J, Bobker S, Simonetto M, Wechsler P, Harpe J, Greer C, Mints G, Salama G, Tsiouris AJ, Leifer D (2020) COVID-19 presenting with ophthalmoparesis from cranial nerve palsy. Neurology 95:221-223

Ellul MA, Benjamin L, Singh B, Lant S, Michael BD, Easton A, Kneen A, Defres A, Sejvar J, Solomon T (2020) Neurological associations of COVID-19. Lancet Neurol 19:767-783. https:// doi.org/10.1016/S1474-4422(20)30221-0

Gutierrez-Ortiz C, Mendez A, Rodrigo-Rey S, San Pedro-Murillo E, Bermejo-Guerrero L, Gordo-Mañas R, de Aragón-Gómez F, Benito-León J (2020) Miller Fisher syndrome and polyneuritis cranialis in COVID-19. Neurology 95:e601-e605. https://doi. org/10.1212/WNL.0000000000009619

Insausti-García A, Reche-Sainz JA, Ruiz-Arranz C, López-Vázquez Á, Ferro-Osuna M (2020) Papillophlebitis in a COVID-19 patient: inflammation and hypercoagulable state. Eur J Ophthalmol 1120672120947591. https://doi.org/10.1177/1120672120947591

Kim SM, Woodhall MR, Kim JS, Kim SJ, Park KS, Vincent A, Lee KW, Waters P (2015) Antibodies to MOG in adults with inflammatory demyelinating disease of the CNS. Neurol Neuroimmunol Neuroinflamm 2(6):e163. https://doi.org/10.1212/ NXI.0000000000000163

Lukiw WJ, Pogue A, Hill JM (2020) SARS-CoV-2 infectivity and neurological targets in the brain. Cell Mol Neurobiol. https://doi. org/10.1007/s10571-020-00947-7
Palao M, Fernández-Díaz E, Gracia-Gil J, Romero-Sánchez CM, DíazMaroto I, Segura T (2020) Multiple sclerosis following SARSCoV-2 infection. Mult Scler Relat Disord 45:102377. https://doi. org/10.1016/j.msard.2020.102377

Ramanathan S, Mohammad S, Tantsis E, Nguyen TK, Merheb V, Fung V, White OB, Broadley S, Lechner-Scott J, Vucic S, Henderson A, Barnett MH, Reddel SW, Brilot F, Dale RC, Australasian and New Zealand MOG Study Group (2018) Clinical course, therapeutic responses and outcomes in relapsing MOG antibody-associated demyelination. J Neurol Neurosurg Psychiatry 89:127-137. https://doi.org/10.1136/jnnp-2017-316880

Shaw VC, Chander G, Puttanna A (2020) Neuromyelitis optica spectrum disorder secondary to COVID-19. Br J Hosp Med 81(9):1-3 https://doi.org/10.12968/hmed.2020.0401

Tsai LK, Hsieh ST, Chang YC (2005) Neurological manifestations in severe acute respiratory syndrome. Acta Neurol Taiwan $14: 113-119$

Zhou S, Jones-Lopez EC, Soneji DJ, Azevedo CJ, Patel VR (2020) Myelin oligodendrocyte glycoprotein antibody-associated optic neuritis and myelitis in COVID-19. J Neuroophthalmol 40:398402. https://doi.org/10.1097/WNO.0000000000001049

Zubair AS, McAlpine LS, Gardin T, Farhadian S, Kuruvilla DE, Spudich S (2020) Neuropathogenesis and neurologic manifestations of the coronaviruses in the age of coronavirus disease 2019: a review. JAMA Neurol 77:1018-1027. https://doi. org/10.1001/jamaneurol.2020.2065

Publisher's Note Springer Nature remains neutral with regard to jurisdictional claims in published maps and institutional affiliations. 